\section{The frequency of clinical manifestations of hypophos- phatemic rickets in patients with therapeutic strategies}

\author{
Leila Kanafi Vahed, ${ }^{1}$ Afshin Arianpur, \\ Mohammad Esmaeili ${ }^{3}$ \\ ${ }^{1}$ Department of Community Medicine, \\ Gilan University of Medical Sciences, \\ Gilan; ${ }^{2}$ Department of Orthopedics, \\ Shohada Hospital, Lahijan, Gilan \\ University of Medical Sciences, Gilan; \\ ${ }^{3}$ Mashhad University of Medical \\ Sciences, Mashhad, Iran
}

\begin{abstract}
The aim of this study was to evaluate the frequency of clinical manifestations of various types of rickets in patients could be effective in therapeutic strategies. A total of eleven child patients ( 7 females and 4 males) were included in the present study. The patients were admitted to the hospital with hypophosphatemic rickets. In our study, the age at the time of diagnosis ranged from 1 to 15 years. Mean age of first presentations at final diagnosis was determined to be 5.5 an 2 years, respectively. Five patients out of 11 patients had showed significant history of vitamin D3 resistance. Family history of similar skeletal problems was reported to be positive in six patients. Furthermore, there were seven relative marriages in parents. Mean value of Gomez index has been revealed as $64 \%$. Mean serum phosphate level was detected as 2.9 $\mathrm{mg} / \mathrm{dL}$, that this level in males $(2.2 \mathrm{mg} / \mathrm{dL})$ was significantly less than females (3.4 $\mathrm{mg} / \mathrm{dL})(\mathrm{P}=0.5)$. Mean serum alkaline phosphatase activity was reached 913 IU/L. Alkaline phosphatase activity in women (1075 IU/L) was significantly lower than that of men (750 IU/L), $(\mathrm{P}=0.5)$. Definitive treatment with oral phosphate and vitamin D3 can prevent complications of the disease and the side effects of unconventional treatments.
\end{abstract}

\section{Introduction}

Rickets is divided into two major categories including calcipenic and phosphopenic. Hypophosphatemia is described as a common manifestation of both categories. ${ }^{1,2}$

Hypophosphatemic rickets is the most common type of rickets that is characterized by low levels of serum phosphate, resistance to ultraviolet radiation or vitamin $\mathrm{D}$ intake. There are several issues involved in hypophosphatemic rickets such as calcium, vitamin D, phosphorus deficiencies. Moreover, other disorder can be associated with its occurrence such as absorption defects due to pancreatic, intestinal, gastric, and renal disorders and hepatobiliary disease. ${ }^{3-5}$ Symptoms are usually seen in childhood and can be varied in severity. Severe forms may be linked to bowing of the legs, poor bone growth, and short stature as well as joint and bone pain. Hypophosphatemic rickets are associated with renal excretion of phosphate, hypophosphatemia, and mineral defects in bones. ${ }^{6,7}$ The familial type of the disease is the most common type of rickets. ${ }^{8}$ In recent years, due to improved nutritional conditions and the addition of vitamin $\mathrm{D}$ to dry milk or the administration of multivitamin, a decrease in nutritional rickets has been observed, while non-nutritional rickets have become relatively more prevalent. Vitamin D-dependent rickets type 2 is a rare genetic disease caused by mutations in the vitamin D receptor and will lead to resistance to the biological effects of calcitriol. This disease is usually resistant to high doses of calcitriol depending on the type of mutation, and the successful treatment of these patients requires modification of hypocalcemia by administering high dose calcium and eliminating the receptor defective route. ${ }^{9-14}$

Given the different clinical, laboratory, and radiological symptoms in children with Rickets, it is necessary to determine the prevalence of these symptoms in children. Furthermore, early detection of rickets can lead to appropriate treatment, due to the simplicity of the treatment and the risk of its complications. Therefore, the frequency of clinical manifestations of various types of rickets in patients can be effective in therapeutic strategies.

\section{Materials and Methods}

The sources of information used were obtained as follow: The patients were admitted to the Ghaem Medical Center and Imam Reza Hospital, where diagnosed with hypophosphatemic rickets and treatmentresistant rickets. These patients have been discharged with a prescription for oral phosphate and vitamin D.

\section{Study population}

The present study was conducted in two sections. In the first part, eleven patients were included, of which four cases were belonged to the admitted patients, and seven cases were outpatients. In the second part of
Correspondence: Afshin Arianpur, Department of Orthopedics, Shohada Hospital, Lahijan, Gilan University of Medical Sciences, Gilan, Iran.

Tel.: +98.9111434497.

E-mail: arian.ortho@yahoo.com

Key words: Hypophosphatemic rickets; skeletal deformities; alkaline phosphatase activity.

Conflict of interest: the authors declare no conflict of interest.

Received for publication: 14 March 2018 Accepted for publication: 20 April 2018.

This work is licensed under a Creative Commons Attribution NonCommercial 4.0 License (CC BY-NC 4.0).

(C) Copyright L.K. Vahed et al., 2018

Licensee PAGEPress, Italy

Clinics and Practice 2018; 8:1072

doi:10.4081/cp.2018.1072

the study, 225 records were extracted from medical records, of which 25 cases were excluded from the study due to a serious deficiency of information (lack of calcium and phosphorus tests) and 200 remaining cases were investigated.

\section{Collecting raw data}

In the first part of the study, an information table was prepared for the better classification of the information and used for one patient. In the second part of the study, an information table was used and information was provided for patients with serum phosphate less than $4 \mathrm{mg} / \mathrm{dL}$. It should be noted that in this section, the hypophosphatemic criteria were used as the first way to screen patients suspected of hypophosphatemic rickets. ${ }^{2}$ Contributing evidence such as positive family history, and vitamin D-resistant rickets were investigated among the patients as well as clinical and laboratory studies, where two suspected patients were identified. The current study was approved by the Ethics Committees of hospitals, Iran.

\section{Variables}

\section{First part of research}

Patients admitted to hospital or outpatient clinics were included who have undergone specific treatment for hypophosphatemic rickets. In these patients, the following cases were extracted and ultimately analyzed: i) sexual distribution of patients: The purpose was to determine the probability of having a sexually transmitted disease in the studied patients; ii) age pattern of patients: In this regard, the age of the 
patient was recorded at the time of first emergence of the disease, as well as the age of the final diagnosis and initiation of specific treatment, the purpose was to determine the delay of diagnosis because one of the assumptions was that our patients were diagnosed late; iii) the main complaints of the patients: a series of symptoms during the first manifestations of the disease and the time of the final diagnosis was obtained, then their association with the sex of the patients and other factors such as the severity of hypophosphatemia were investigated; iv) parent's cousinship: parent's cousinship has been determined to facilitate the emergence of autosomal recessive inheritance. Family history problems with a patient such as short stature, skeletal deformity, and vitamin D-resistant rickets were also considered; v) the history of drug use, the frequency of vitamin D3 injection was determined with a poor diagnosis of nutritional rickets for determining the amount of inappropriate treatments. Because one of the preconceptions was the probability of inappropriate treatment; vi) the growth status: this condition of patients has been investigated for short stature, which is one of the most common manifestations of the disease. In this regard, in addition to recording the height and weight of patients, Gomez, Waterlow and BMI criteria were used to assess malnutrition and its severity; vii) laboratory findings: as far as possible, the results were recorded in the relevant table. Particularly FEP was calculated, which is a good diagnostic criterion; viii) imaging findings: the results of the patient's bone radiography, especially the wrist, were examined as much as possible, and in some cases, renal imaging was also carried out. The aim of the study was to investigate the possible side effects of unusual use of vitamin D (such as nephrocalcinosis). Because one of the presuppositions was that our patients are likely to have complications due to delayed diagnosis and treatment; ix) therapeutic methods: therapeutic methods and results of subsequent referrals of patients with regard to the progression of recovery and response to combination therapy were evaluated, since it is a reliable proof of the accuracy of the final diagnosis.

\section{Second part of the study}

In this section, patients with rickets diagnosis were evaluated and the hypophosphatemia ( $\mathrm{Pi}<4 \mathrm{mg} / \mathrm{dL}$ ) was used as the first criteria for screening patients suspected to hypophosphatemic rickets. The following criteria were recorded in these patients: i) age and sex; ii) signs such as short stature, and smooth bowing; iii) family history, especially family history of short stature or resistance to rickets therapy; iv) the history of the drug use, especially previous use of vitamin D compounds and patients with vitamin-D resistant rickets; v) growth criteria, especially short stature as the most common symptom of hypophosphatemic rickets; vi) the results of blood biochemistry, 24-h urine, ABG and other useful tests were obtained, which were especially noted for FEP; vii) radiological evidence and the possibility of typical symptoms of hypophosphatemic rickets in radiographs; viii) recommended treatment in these patients.

\section{Results and discussion}

\section{Statistical analysis of variables (first part of the study)}

Overall, 11 patients were evaluated in this study, of which 7 (63.6\%) girls and 4 (36.4\%) were boys. Z-Test showed that there was no statistically significant difference between girls and boys in terms of the prevalence $(\mathrm{P}=0.05)$. The patient's age ranged from 1 and 15 years old at the time of the final diagnosis. The mean age of the patients was 5.5 years, followed by the median age (4 years) and the mode age (4 years). The mean ages of the girls and boys with the final diagnosis were determined to be 5.5 and 6.2 years, respectively. With regard to the t test, there was no statistically significant difference in the mean age of the patients at the time of final diagnosis $(\mathrm{P}=0.05)$.

Given the formula d' in the classified information, the variance and standard deviation of the age were calculated (12.96 and 3.6, respectively). Regarding the average age, our information in terms of age had a normal distribution (Gaussian distribution) $(\mathrm{P}=0.05)$. By calculating the coefficient of age variation in two sexes (girls 54\% and boys $29 \%$ ), it was revealed that the age distribution of the affected girls is more than that of boys.

The patient's age ranged from 1 to 6 years old at the time of the onset of the first symptoms and referral to the physician (mean: 24 months, median: 13.5, mode: 12 months). The t-test was used to compare the possible difference between the mean age of girls and boys when the first symptoms occurred. The mean age of girls and boys was 26.5 and 12 months after the first symptoms, respectively. After calculating variance and standard deviation, despite the apparent difference, there was no significant difference in mean age of initial incidence among the two sexes $(\mathrm{P}=0.05)$.

The delay from the onset of the first symptoms and referral to doctors was recorded, as well asthe time for the final diagnosis and combination therapy of phosphate and active vitamin D. This delay ranged from 0 to 6 years. The average delay was then calculated to be 3 years for all patients, following a mean delay in girls (3.3) and a mean delay in boys (1.7). There was no statistically significant difference in diagnostic delay between boys and girls ( $\mathrm{P}$ $=0.05)$.

Vitamin D-resistant rickets was seen in 9 cases $(82 \%)$ with vitamin D injection (3 to 50 times). Family history of similar bone problems was reported in six $(55 \%)$ positive patients, and the rest did not have such a history. Regarding the parent's cousinship, this was seen in seven cases (64\%). One case had no parent's cousinship, while it was not mentioned for three cases where excluded from the statistics.

With regard to growth indices, short stature was found in all cases.

Gomez criteria were calculated in nine cases and two cases were excluded. The Gomez Changes ranged from $34 \%$ to $87.5 \%$ (mane Gomez: 64\%, mode: 64\%). Failure to thrive (faltering growth) was to evaluate severe FTT (Failure to thrive; with Gomez score less than $60 \%$ ), the Z-test was used for both sexes, where the findings showed that there was no significant difference between boys and girls $(\mathrm{P}=0.05)$.

To evaluate Gomez's mean difference in both sexes, t-test was applied which did not show significant statistical difference. The Waterlow score was measurable in nine cases and two cases were excluded. The Waterloo score ranged from 139 to $80 \%$. The Waltlow score were determined in seven patients $(78 \%)$ above $90 \%$. To determine the mean Waterlow difference between girls and boys, t-test was used (mean of Waterloo for girls: $98.3 \%$ and $10.5 \%$ for boys). There was no statistically significant difference between the two groups $(\mathrm{P}=0.05)$.

The results showed that the serum calcium level was $8.5-12.5 \mathrm{mg} / \mathrm{dL}$, and the normoclcemia was reported in three cases only. Seven out of 8 patients had more than 8.5 $\mathrm{mg} / \mathrm{dL}$ of calcium $(87 \%)$.

The levels of inorganic phosphate were determined to be 1.3-4 mg/dL; hypophosphatemia was also reported in three cases (mean serum phosphate: $2.9 \mathrm{mg} / \mathrm{dL}$ and mode: $3 \mathrm{mg} / \mathrm{dL}$ ). Using statistical t-test, there was a significant difference in the mean of serum phosphate in girls and boys (3.4-2.2) in our patients $(\mathrm{P}=0.5)$. In three cases, phosphate reduction was not mentioned, which was eliminated from the statistics. Serum alkaline phosphatase levels were also studied, where there had been 3 cases of high ALP, while the rate was 
reported to be $282-26,685 \mathrm{U} / \mathrm{L}$ in other cases (mean: 93 and mode: 809). The mean of ALP in girls and boys was 1075 and 750 $\mathrm{m}$ respectively. This difference was found to be statistically significant using the $t$ test $(\mathrm{P}=0.5)$.

Serum sodium levels were not mentioned in three cases and the remaining cases ( 8 patients) showed normal levels between 135-145 Meq/l. Potassium levels were only mentioned in 8 cases where 2 cases showed hypokalemia $(\mathrm{K}<3.5 \mathrm{meq} / \mathrm{L})$. Moreover, BUN and $\mathrm{Cr}$ were detected in seven patients, where all of whom were normal.

The rate of phosphate excretion (FEP) was slightly evaluated in five patients and the increase in FEP was noted without mentioning the absolute level of FEP in three patients, while this rate was not recorded in 3 cases. In feasible cases, FEP was determined to be between $30 \%-55 \%$, with an average of $44 \%$ indicating a sharp increase in phosphate leakage. On the other hand, $\mathrm{ABC}$ was performed in 7 cases, where showed normal ABC, while two showed metabolic acidosis. However, 2 patients had no any documentation in terms of $\mathrm{ABC}$.

The main complaints of the patients (73\%; 8 cases) were reported short stature and deficiency of growth or delayed walking (27\%).

Considering the symptoms of patients during presentation and final diagnosis, deficiency of growth was observed in 11 cases $(100 \%)$, followed by delayed walking ( 7 cases; $63.6 \%$ ), bone deformity ( 8 cases; $73 \%)$ and delayed walking with deformity ( 5 cases; $45.5 \%$ ). Furthermore, the coexistence of three signs of deficiency of growth, delayed walking and deformity was noted in 5 cases $(45.5 \%)$ during presentation and final diagnosis. To compare the prevalence rate of each symptom in either sex or the presence of a combination of three signs in each of the girls and boys groups, the $\mathrm{z}$ test was used. The results revealed that there was no significant difference in the prevalence of symptoms between the two sexes $(\mathrm{p}=0.05)$. Due to the lack of sufficient numbers of samples, it was not statistically possible to assess the gender relationship with the prevalence of the three mentioned symptoms.

\section{The second part of the study: evaluation of patients with rickets}

In this study, 200 cases of patients admitted in Ghaem and Imam Reza centers with Rickets diagnosis were evaluated between $65-75$ years. A total of 86 cases (43\%) showed a decrease in inorganic phosphate levels $(\mathrm{P}<4 \mathrm{mg} / \mathrm{dL})$. Of these 86 patients, 2 cases of rickets were identified with a history of vitamin D resistance. Total serum phosphate was found to be less than $4 \mathrm{mg} / \mathrm{dL}$ in 86 cases, of which $52(60 \%)$ were male and $34(40 \%)$ were female. The most common cause of hospitalization of these patients was seizures associated with fever in the first place, followed by skeletal symptoms, and generalized seizures without fever, pneumonia, and sepsis. The age ranged from 2 and 14 years old.

\section{Overall outcomes and suggestions}

In the first part, there was no statistically significant difference in the prevalence of sex in our patients. Patients aged 15-1 years old had no significant difference in mean age of girls and boys. The first symptoms if disease were seen between the ages of 1 and 6 (average, 2 years), where did not have a significant difference in sexes. The average latency of the final diagnosis was determined at the time of the first symptoms of the disease for about 3 years. 9 out of 11 patients who had previously experienced frequent injections of vitamin D3, while showing a lack of clinical and radiological response. Six cases demonstrated a positive family history.

All patients had suffered from short stature, skeletal problems and delayed walking. The serum calcium level in all patients was higher than $8.5 \mathrm{mg} / \mathrm{dL}$, while the average serum phosphate was 2.9 $\mathrm{mg} / \mathrm{dL}$, which is in agreement with previous studies The statistical evaluation showed that the severity of hypophosphatemia in male patients was higher than that of girls $(\mathrm{P}=0.5)$. The mean serum alkaline phosphatase level was $913 \mathrm{mg} / \mathrm{dL}$, which was significantly higher in girls than males $(\mathrm{P}=0.5)$. The levels of sodium, potassium, BUN, and creatine were in the normal range. $\mathrm{ABG}$ results were only indicative of metabolic acidosis, which was probably due to secondary tubulopathy for repeated injections of vitamin D3 and was accompanied by hypokalemia and nephrocalcinosis in sonography. Statistical tests showed that the severity of the disease (i.e., the variability of symptoms, premature appearance) and the severity of rickets (i.e., short stature and deformity) had no statistical relation with the severity of hypophosphatemia, which is consistent with the results of other researchers. In the second part of the study, evaluation of 200 records revealed two possible cases of hypophosphatemic rickets using serum phosphate standard less than 4 $\mathrm{mg} / \mathrm{dL}$ (hypophosphatemia) as the first screening of patients with Vitamin D-resistant rickets. Regarding the above findings, it seems that we have been able to achieve some of our goals and preconceptions. Our attention has been drawn to the long delay since the onset of the first manifestation of the disease until reaching the final diagnosis, which had led to a progressive destructive disease in most patients and the development of problems such as severe short stature, multiple bone deformities as well as medical conditions linked to inability to move. On the other hand, this has led to frequent and misleading injections of vitamin D3; as results, complications such as nephrocalcinosis and secondary tubulopathy have been observed.

\section{Conclusions}

In summary, considering all the diagnostic limitations and laboratory facilities, as well as the financial weakness of patients and other problems, it seems that additional diagnostic measures, such as 24-h management, FEP determination, PTH measurement, ABG, etc. should be performed. Because timely detection after the onset of the first symptoms of a disease and the definitive treatment with oral phosphate and vitamin D3 can prevent complications of the disease and the side effects of unconventional treatments.

The following issues should be taken in to consideration for dealing with patients: i) history of resistance to vitamin D treatment in children and his family; ii) family history of problems such as short stature or skeletal deformity in parents or a family relative with rickets; iii) the children who are diagnosed with the index case and are suffering from rickets where did not receive normal clinical and radiological responses to normal rickets nutritional treatment; iv) the clinical presentation of smooth bowing of the limbs, enteropathy, lack of myopathy, etc.; v) typical radiographic symptoms of hypophosphatemic rickets such as coarse trabeculation, cortical thickening, evidence of rickets without secondary hyperparathyroidism, etc.

\section{References}

1. Jagtap VS, Sarathi V, Lila AR, et al. Hypophosphatemic rickets. Indian J Endocrinol Metabol 2012;16:177-82.

2. Tiosano D, Hochberg Z. Hypophosphatemia: the common denominator of all rickets. J Bone Miner Metab 2009;27:392-401.

3. Bielesz B, Klaushofer K, Oberbauer R. Renal phosphate loss in hereditary and acquired disorders of bone mineralization. Bone 2004;35:1229-39.

4. Sahay M, Sahay RK. Refractory rickets 
in the tropics. J Pediatr Endocrinol Metab 2010;23:597-601.

5. Xu C, Ma C, Bai Y. A pediatric hypophosphatemic rickets on MRI, (99m)Tc-MDP bone scan and (18)FFDG PET/CT. Hell J Nucl Med 2017;20:93-6.

6. Fujiwara M, Namba N, Ozono K, et al. Treatment of hypophosphatemic rickets with phosphate and active vitamin D in Japan: a questionnaire-based survey. Clin Pediatr Endocrinol 2013;22:9-14.

7. Yamamoto T, Michigami T, Aranami F, et al. Hereditary hypophosphatemic rickets with hypercalciuria: a study for the phosphate transporter gene type IIc and osteoblastic function. J Bone Miner
Metab 2007;25:407-13.

8. Jagtap VS, Sarathi V, Lila AR, et al. Hypophosphatemic rickets. Indian $\mathrm{J}$ Endocrinol Metab 2012;16:177-82.

9. Shafeghati Y, Momenin N, Esfahani T, et al. Vitamin D-dependent rickets type II: report of a novel mutation in the vitamin D receptor gene. Arch Iran Med 2008;11:330-4.

10. Hawa NS, Cockerill FJ, Vadher S, et al. Identification of a novel mutation in hereditary vitamin D resistant rickets causing exon skipping. Clin Endocrinol (Oxf) 1996;45:85-92.

11. Ghazi AA, Zadeh-Vakili A, Zarif Yeganeh M, et al. Hereditary vitamin D resistant rickets: clinical, laboratory, and genetic characteristics of 2 Iranian siblings. Int $\mathrm{J}$ Endocrinol Metab 2017;15:e12384.

12. Soni SS, Adikey GK, Raman AS. Vitamin D dependent rickets type II: late onset of disease and response to high doses of vitamin D. Saudi J Kidney Dis Transpl 2008;19:796-8.

13. Azemi M, Berisha M, Ismaili-Jaha V, et al. Vitamin D - dependent rickets, type II case report. Mater Sociomed 2014;26:68-70.

14. Pang Q, Qi X, Jiang Y, et al. Clinical and genetic findings in a Chinese family with VDR-associated hereditary vitamin D-resistant rickets. Bone Res 2016;4:1038. 